\title{
Taoist Medicine
}

\author{
Zhang Ping, $M D$ | ORCID: 0000-0003-0346-2537 \\ General department and Geriatric Center, Xiyuan Hospital, \\ China Academy of Chinese Medicine Sciences, Beijing, China \\ pingpingo9291103@126.com
}

\author{
Zhang Dong, $M D$ \\ Cardiovascular center, Xiyuan Hospital, China Academy of Chinese \\ Medicine Sciences, Beijing, China \\ yixinfang9@163.com
}

\begin{abstract}
Traditional Chinese medicine originated from Taoist thought in the pre-Qin period of China, especially the classic "Yellow Emperor's Internal Classic of Chinese medicine", while Chinese Taoism also originated from pre-Qin Taoist thought. The representative figure of pre-Qin Taoist thought is Lao Tzu, and his work "Tao Te Ching" is used as a reference Representative, as a Chinese religion pursuing cultivation to become a god, Chinese Taoism respects Lao Tzu as the supreme old monarch and regards him as the leader, and uses Lao Tzu's “Tao Te Ching” as a classic. Therefore, Traditional Chinese medicine and Taoism share the same origin. Taoism believes that in order to become immortal, diseases must be eliminated. Therefore, Taoism in turn uses Traditional Chinese medicine to form a unique Taoist medicine, which is recorded in the Taoist classic "Tao Zang". There are many prescriptions derived from Taoism and Traditional Chinese medicine.
\end{abstract}

\section{Keywords}

Taoism - Taoist medicine - psychotherapy - traditional Chinese medicine

Taoism is rooted in the soil of Chinese culture. In the process of long-term development and melting, it not only inherits Taoist thoughts, but also includes various thoughts of Mohist, Military and Legalist. Therefore, Taoism 
integrates many Chinese folk cultures, and integrates numerous and confusing philosophies, myths and witchcraft into a religious system which is the only religion born and raised in China. ${ }^{1}$ Taoism has gradually formed a unique Taoist medicine in the long time and development process. The definition of Taoist medicine in Encyclopedia of China Religious Volume is the following: Taoist medicine is the medical knowledge and technology accumulated by Taoism in the process of internal cultivation and external cultivation in pursuit of immortality, inheritance and absorption of Chinese traditional medical achievements. It includes taking medicine, external medicine, internal medicine, guiding technique (also named Dao Yin), elixir and spells with witch doctor color, etc. It is related to and different from Chinese traditional medicine, and its essence of medicine and pharmacology is an integral part of Chinese medicine. It is a diversified medical model integrating physiological therapy, belief therapy and psychotherapy. ${ }^{2}$

Taoist medicine originated from the philosophical thoughts in the preQin period, especially the Yi-ology thoughts and Taoist thoughts in the preQin period. Its theoretical basis includes the theory of Yin and Yang and Five Elements, the principle of induction between human and nature, and the monism of Qi. ${ }^{3}$ The Yi-ology expounds the dynamic and static changes of Yin and Yang in everything in the world. Yin and Yang theory and Five Elements theory are typical important philosophical thoughts in ancient China. In the "Classic of Great Peace", an early Taoist classic, the thought of Yin-Yang and Five Elements was also absorbed, which was used to examine the nature of everything in the world and explain the truth of meditation. Another Taoist classic, "Zhou Yi Shen Tong Qi", uses hexagrams and lines to match the Yin and Yang and the Five Elements to lay the theoretical foundation of Taoist golden alchemy. "Tao Te Ching", which is the Taoism classic works of pre Qin, says that: ${ }^{4}$ Tao produces One, the One produces Two (Yin and Yang), and the Two produces Three (Yin, Yang and the exchange between Yin and Yang), then the Three generates all things. All things leave behind them the Obscurity (out of which they have come), and go forward to embrace the Brightness (into which they have emerged), while they are harmonised by the Breath of Vacancy. "Huai nanzi" says that Tao begins with One, One can be divided into Yin and Yang, Yin and Yang are harmonious and everything is born. "One Qi" believes that the same "Qi" penetrates all things in the world. Qi is the matrix of all life,

1 See Hou Bing, Exploring the Relationship between TCM Academic and Taoist Culture.

2 See China Encyclopedia Editorial Committee, Encyclopedia of China, Religious Volume.

3 See Li Yi, Brief explanation of Taoist medicine, p. 58.

4 See Lao Tzu, The Eternal Tao Te Ching. 
and Yin and Yang are sympathetic to produce all things. People are born if they have qi, and die if they do not have it. The main methods in preserving health and treating diseases are nourishing and regulating qi. These philosophical thoughts laid the foundation of Taoist medicine, which deeply influenced Taoist doctors' views on nature, human body and life, and were embodied in various medical practices of Taoist medicine.

Taoist physicians constantly put forward new theories, both rom the perspective of medical theory and clinical practice, and at the same time summarized and created many new treatment methods. In the process of the development and dissemination of Taoism, no matter whether for others or for themselves, it is necessary to treat diseases, and monks are duty-bound to treat diseases. Taoist doctors attach great importance to the medical needs of lower-class patients. Many Taoist doctors also write books to facilitate the treatment needs of poor people. ${ }^{5}$ Ge Hong, a Taoist physician and Taoist priest in the Eastern Jin Dynasty (317-420 AD), is a typical representative figure. He pays great attention to medicine facing the bottom of society. Most of the drugs recorded in the book are "cheap medicinal materials" which are found everywhere and easy to collect. He also advocated that acupuncture and moxibustion should be used in combination with medicine, emphasizing that the position of moxibustion should only be measured on its scale, and not on the accurate acupuncture points. These treatment methods and drugs reflect the characteristics of "simplicity, convenience and cheapness", which meet the medical needs of ordinary people. Sun Simiao, a Daoist doctor in Tang Dynasty, who is regarded as the King of Medicine, also embodied the spirit of "treating medicine as benevolence" in "Great Doctor Sincerity", which reflected the medical view of treating all living beings equally in the process of applying medicine to save people, and showed that Taoist doctors have a broad mind of helping the world.

Taoist medical system includes not only herbal medicine, decoction, ointment, acupuncture of traditional Chinese Medicine, etc. It also includes guiding technique, massage, valley-clearing, beauty-care, qi-therapy, etc. Then there are spells, medicine labels, prayers, sacrifices, fasting and other therapies which have strong religious belief characteristics and diversified treatment methods. Many Taoist doctors have written medical and pharmaceutical works during the period of practicing medicine and monasticism, such as Tao Hongjing's "Materia Medica Annotations" and Ge Hong's "Elbow Reserve Emergency Recipe". "Materia Medica Annotations" pioneered the classification of drugs according to the nature of the drugs, and "Elbow Reserve Emergency

5 See Xu Yan, Summary of Ancient Taoist Doctors. 
Recipe" provided people at that time with a simple and easy-to-operate first aid method, saving countless people, and still gives us medical enlightenment and reference. Acupuncture is also a common method used by Taoist doctors. $\mathrm{Bao} \mathrm{Gu}$, a Taoist doctor, is a famous acupuncturist. Her deeds of practicing medicine and saving people are widely spread in many places in the south. Later generations also developed new therapies such as midnight-noon ebbflow, Linggui Eight Methods, Feiteng Eight Methods and Taiyi Shenzhen.

Taoism is characterized by refining alchemy medicine in pursuit of immortality. Alchemy can be divided into external alchemy and internal alchemy. External alchemy takes vegetation, cinnabar, sulfur, copper and mercury as important raw materials, and is supplemented with nitrate, lime, white salt and arsenic, which are collectively referred to as "Hardware Eight Stones". Some of the refined external alchemy medicines have the functions of killing insects and treating syphilis, and are used in surgery to remove putrefaction and promote granulation. Although it is impossible to achieve the goal of immortality, this pursuit has also promoted the development of pharmaceuticals, metallurgy and chemistry. The inner alchemy takes the human body as the "furnace pot", the qi as the medicine, the heart as the god, and the god as the fire. Under the strict control of man-made spiritual consciousness, the essence and Qi secreted by the human body are cultivated through the whole body circulation by using the driving force of vitality in the human body, so that the essence, Qi and spirit are condensed into "holy fetus" or "pill". Through the cultivation of vitality of life, it can be refined into a "golden pill", hidden in the life gate and running the whole body, and constantly warming it, so that it can exert infinite vitality and potential of life and achieve the wonderful realm of immortality. ${ }^{6}$ Taoist meditation is a kind method to cultivate, and it can induce a distinct and reversible mental state that provides insights into brain correlates of consciousness. ${ }^{7}$

Guiding technique, massage technique, and exhaling and convincing are also widely used by Taoist doctors. Guiding technique is a form of exercise combining physical movements, mental focus, and breathing originated in ancient China. ${ }^{8}$ Massage method is included in Tao Hongjing's "Yang Xing Yan Ming Lu”, Sun Simiao's “Thousand Golden Preions” and other Taoist scriptures in Taoist Collection. There are more than 10 kinds of works about massage in "Dao Zang", and massage techniques and positions are also described in detail.

6 See Gai Jianmin, Academic History and Research Status of the Relationship between Taoism and TCM.

7 See Jao T. et al., Large-Scale Functional Brain Network Reorganization During Taoist Meditation.

8 See Chen X. et al., Dao Yin (a.k.a. Qigong). 
$\mathrm{Fu} \mathrm{Qi}$ is a kind of breathing exercise. At a specific time, breathing exercises are carried out in a specific way, such as when the sun rises, facing the sun, accompanied by knocking teeth, swallowing qi, swallowing jin, chanting wishes, etc., to promote qi and cure diseases, and later generations also developed the sixcharacter tactic method, etc. ${ }^{9}$

Taoist medicine is very good at using psychotherapy. Taoistic concepts of mental health stress the transcendence from self and secularity, the dynamic revertism of nature, integration with nature and the pursuit of the infinite. ${ }^{10}$ Chinese Taoist cognitive therapy (СтСт) is a culture-oriented psychological therapy for mental well-being. Some studies have been proved to help reduce depressive symptoms in Chinese adults, including patients with chronic physical diseases and clinical or non-clinical depression. ${ }^{11}$ In addition, Стст also has effects on the treatment of anxiety disorders and post-stroke depression. ${ }^{12}$ In fact, Taoist doctors attach importance to the "God" of the human body. Among the various methods of psychotherapy, spell therapy is a unique and controversial treatment method in Taoist medicine. This method is related to the attraction of early Taoist missionary to the congregation, and has a strong color of witch doctor. As far as "Fu water" is concerned, "Fu" refers to the words used to pray for gods and exorcise demons. Early Taoism believed that putting paper printed with "Fu" into water could exorcise demons and remove diseases after drinking. In addition to drug treatment factors in the treatment of spells therapy, it should be largely attributed to the psychological medical effect of the spells therapy in the treatment of diseases.

Taoist medicine also attaches great importance to keeping in good health. Taoism always advocates the ideas of "valuing people and valuing health" and "my life is in my hands", and emphasizes the concept of "preventing diseases before treatment". In the process of discussing the treatment of different diseases, especially intractable diseases, chapter 5 in the medical classic "Yellow Emperor's Internal Classic of Chinese medicine" mentioned: "Therefore, the best treatment is to treat from fine hair, to muscle, to pulse, to six fu organs,

9 See Li Jinxi, On the Homology and Penetration of Taoism and Chinese Medicine.

10 See Kam-shing Yip, Taoism and its impact on mental health of the Chinese communities.

11 See Yudan Ding et al., Chinese Taoist Cognitive Therapy for Symptoms of Depression and Anxiety in Adults in China, p. 769; Huang Qingyuan/Li Jianguo, Clinical Effect of Chinese Taoist Cognitive Therapy.

12 See Li Meizhi et al., Effect of Chinese Taoist Cognitive Therapy on Mental Health of Family Members of Neurosis Patients; Li Xian/Chen Hao, Combined Taoist Cognitive Psychotherapy with Hypnotherapy; Liu Yan, Effects of Chinese Taoist Cognitive Therapy; Zhang Gaofeng, Effects of Chinese Taoist Cognitive Therapy on Social Function. 
and the five internal organs". ${ }^{13}$ Taking obeying nature as the general principle of keeping in good health, and taking both form and spirit as the concept of keeping in good health, we conform to the natural rhythm and both form and spirit. Good health needs to start from the aspects of diet, daily life, exercise and emotion. Among them, emotion is the spiritual, psychological and emotional level, which occupies the primary position among all the elements of health preservation.

In addition to the above-mentioned various Taoist medical treatment methods, Taoist doctors attach great importance to food maintenance and put forward the viewpoints of "having less appetite, not eating hot but not cold, eating lightly, eating slowly, taking a hundred steps after meals, rubbing one's hands and rubbing one's belly", which have had a great impact on people's daily life so far, and have also confirmed the scientific validity of these viewpoints in practice. ${ }^{14}$

Today, Tu Youyou, a great researcher at China Academy of Chinese Medical Sciences, was inspired by "Elbow Reserve Emergency Recipe", written by Ge Hong. She invented artemisinin and won the Nobel Prize in Medicine. On the basis of all the classic works, like the Taoist classic "Tao Te Ching", the medical classic "Yellow Emperor's Internal Classic of Chinese medicine", the philosophical and divination classic "Zhou Yi" which is closely related to Taoism, Dr. Zhang Dong, who also works at China Academy of Chinese Medical Science, founded the "Yuanqi Shenji method" and created two important decoctions, Guiyi Decoction and Guanfu Decoction, which have achieved very good effects in the treatment of various clinical diseases. Up to now, some spells and scribbles in Taoist medicine still play a role in curing diseases among the people.

To sum up, Taoist medicine takes life and disease as the core, fellows the Taoist view of life and disease prevention and treatment, and pays attention to the application of various technical means created and invented by physicians under the influence of this concept. ${ }^{15}$

\section{Biography}

Zhang Ping, female, deputy chief physician, MD, master supervisor. She graduated from Beijing University of traditional Chinese medicine in 2012. And she has learned from many experiences and skills from several famous тсм

13 See Wang Bing, Yellow Emperor's Canon: Internal Medicine.

14 Wang Minghui et al., Food and Drug Therapy in Taoist Medicine.

15 See Xu Wei, Tao Hongjing Taoist Medical Research. 
masters. Now the main research direction is focus on the treatment of elderly hypertension complicated with mild cognitive impairment.

Zhang Dong, male, chief physician, MD, the second batch of postdoctoral teachers of the State Administration of traditional Chinese medicine. He graduated from Beijing University of traditional Chinese medicine in 1993. The fourth batch of national famous and old academic successors of traditional Chinese medicine are taught by Professor Weng Weiliang. Young and middleaged Chinese doctors of the Chinese Academy of traditional Chinese medicine. The fourth generation of Yanjing medicine. He is the founder of pre-Qin traditional Chinese medicine.

\section{Bibliography}

Chen X./Cui J./Li R./Norton R./Park J./Kong J./Yeung A.: Dao Yin (a.k.a. Qigong): Origin, Development, Potential Mechanisms, and Clinical Applications, in: Evidence-Based Complement Alternative Medicine 21 (10/2019), pp. 1-11.

Gai Jianmin: Academic History and Research Status of the Relationship between Taoism and TCM, in: World Religious Culture 5 (2020), pp. 22-28.

Hou Bing: Exploring the Relationship between TCM Academic and Taoist Culture, in: Xinjiang Traditional Chinese Medicine 3 (2002), pp. 1-31.

Huang Qingyuan/Li Jianguo: Clinical Effect of Chinese Taoist Cognitive Therapy in Treating Major Depressive Disorder in 35 Cases, in: Chinese Med. Gansu 18 (2005), pp. 18-19.

Jao T./Li C.W./Vértes P.E./Wu C.W./Achard S./Hsieh C.H./Liou C.H./Chen J.H./ Bullmore E.T.: Large-Scale Functional Brain Network Reorganization During Taoist Meditation, in: Brain Connect 6 (2/2016), pp. 9-24.

Kam-shing Yip: Taoism and its Impact on Mental Health of the Chinese Communities, in: International Journal of Social Psychiatry 50 (1/2004), pp. 25-42.

Lao Tzu: The Eternal Tao Te Ching. Beijing: China Intercontinental Press 2014.

Li Jinxi: On the Homology and Penetration of Taoism and Chinese Medicine. Sichuan: Sichuan University 2004.

Li Meizhi/Guo Tiansheng/Wang Jinai/Tan Benteng/Long Haowen/Tan Meijuan/ Wang Guoqiang: Effect of Chinese Taoist Cognitive Therapy on Mental Health of Family Members of Neurosis Patients, in: Asia-Pacific Tradition Med. 4 (2008), pp. $46-47$.

Li Xian/Chen Hao: Combined Taoist Cognitive Psychotherapy with Hypnotherapy in the Treatment Anxiety Disorders, in: Tibetan Medicine (2009), pp. 6-7.

Li Yi: Brief explanation of Taoist medicine, in: Chinese Religion 7 (2020), p. $5^{8}$. 
Liu Yan: Effects of Chinese Taoist Cognitive Therapy Combined with Acupuncture in the Treatment of Post-stroke Depression, in: Chinese Journal of Integrative Medicine on Cardio-/Cerebrovascuiar Disease 7 (2009), pp. 986-987.

The Editorial Committee China: Encyclopedia of China, Religious Volume. Beijing: Encyclopedia of China Publishing House 2015.

Wang Bing: Yellow Emperor's Canon: Internal Medicine. Taos: Redwing Book Co 2001.

Wang Minghui/Jiang Shisheng/Wang Fenglei: Food and Drug Therapy in Taoist Medicine, in: Research on Medicinal Diet and Diet Therapy 2 (1999), p. 4 et seq.

Xu Wei: Tao Hongjing Taoist Medical Research. Hunan: Hunan Normal University 2019. Xu Yan: Summary of Ancient Taoist Doctors. Lanzhou: Lanzhou University 2009.

Yudan Ding/Li Wang/Jindong Chen/Jingping Zhao/Wenbin Guo: Chinese Taoist Cognitive Therapy for Symptoms of Depression and Anxiety in Adults in China: A Systematic Review and Meta-Analysis, in: Front Psychology 11 (2020).

Zhang Gaofeng: Effects of Chinese Taoist Cognitive Therapy on Social Functions for Patients with Major Depressive Disorder, in: Henan Institute of Sci. Technol. 5 (2013), pp. 111-113. 